\title{
END-TO-END CONSTRUCTION FOR THE ALLEN-CAHN EQUATION IN THE PLANE
}

\author{
MICHA£ KOWALCZYK, YONG LIU, FRANK PACARD, AND JUNCHENG WEI
}

\begin{abstract}
In this paper, we construct a wealth of bounded, entire solutions of the Allen-Cahn equation in the plane. The asymptotic behavior at infinity of these solutions is determined by $2 L$ half affine lines, in the sense that, along each of these half affine lines, the solution is close to a suitable translated and rotated copy of a one dimensional heteroclinic solution. The solutions we construct belong to a smooth $2 L$-dimensional family of bounded, entire solutions of the Allen-Cahn equation, in agreement with the result of [3] and, in some sense, they provide a description of a collar neighborhood of part of the compactification of the moduli space of $2 L$-ended solutions for the Allen-Cahn equation. Our construction is inspired by a construction of minimal surfaces by M. Traizet [12].
\end{abstract}

\section{INTRODUCTION}

This paper deals with the construction of bounded solutions of the Allen-Cahn equation

$$
\Delta v+v-v^{3}=0
$$

which are defined in $\mathbf{R}^{2}$.

We recall that, for any unit vector $\mathbf{a} \in \mathbf{R}^{2}$ and for any $b \in \mathbf{R}$, the function

$$
\mathrm{x} \mapsto \tanh \left(\frac{\mathrm{a} \cdot \mathrm{x}+b}{\sqrt{2}}\right),
$$

is a solution of (1.1) whose nodal set is the affine line defined by $\mathrm{a} \cdot \mathrm{x}+b=0$. These solutions are usually referred to as the heteroclinic solutions of (1.1) and they play a central role in the study of this semilinear elliptic equation. For example, the validity of De Giorgi's conjecture in dimension 2 ensures that any bounded, entire solution of (1.1) which is monotone in one direction must be one of the heteroclinic solutions [5]. Heteroclinic solutions do not exhaust the set of bounded, entire solutions of (1.1) and in fact there are many solutions of (1.1) which are not monotone in any direction. A classical example is the so called saddle solution, whose nodal set consists of two orthogonal straight lines [2]. This example - and its construction - generalize to solutions with dihedral symmetry [1]. In [4] it is proven that there exist solutions whose nodal lines are almost parallel straight lines which are related to solutions of a Toda system.

1991 Mathematics Subject Classification. 35J61.

Y. Liu is partially supported by NSFC grant 11101141 and the project sponsored by SRF for ROCS, SEM, M. Kowalczyk is partially supported by Chilean research grants Fondecyt 1090103, Fondo Basal CMM-Chile, Project Anillo ACT-125 CAPDE, and a MathAmSud project NAPDE, F. Pacard is partially supported by ANR-11-IS01-0002 grant and a MathAmSud project NAPDE, and J. Wei is partially supported by General Research Fund from RGC of Hong Kong. 
All the solutions constructed in the above references share the common property that their structure at infinity is easy to describe. Indeed, away from a compact set, their nodal set consists of a finite number of disjoint curves which are asymptotic to half affine lines. Moreover, along any of these affine lines $\Lambda$ the solution is asymptotic to a copy of a heteroclinic solution whose nodal set is precisely given by $\Lambda$. These affine lines are called the ends of the solution and, if $2 L$ is the number of ends - which is necessarily an even number - such solutions are called $2 L$-ended solutions of the Allen-Cahn equation. We refer to [3] for precise definitions of these notions. Therefore, the heteroclinic solutions are models at infinity for $2 L$-ended solutions. The set of $2 L$-ended solutions of the Allen-Cahn equation is denoted by $\mathcal{M}_{2 L}$. It is proven in [3] that, near any nondegenerate element, $\mathcal{M}_{2 L}$ is a $2 L$ dimensional smooth (real analytic) manifold. Finally, let us mention that the Morse index of any element of $\mathcal{M}_{2 L}$ is finite [9].

When $L=2$, thanks to [6], [9] and [10], we have a rather complete description of the set of 4-ended solutions $\mathcal{M}_{4}$. This description is of particular importance in the present paper and we will return to it in Section 3. Building on this, in this paper, we explain how to construct a wealth of new solutions of (1.1) by gluing together several 4-ended solutions of (1.1) along their ends starting from a given set of affine lines.

\section{Statement of the Results}

Given an integer $L \geq 2$, we consider a finite number of affine lines $\Lambda_{1}, \ldots, \Lambda_{L}$ in the plane and assume that not two of these lines are parallel and not three of them intersect at a common point. If this is the case, we will say that the set of affine lines is admissible.

The union of $\Lambda_{1}, \ldots, \Lambda_{L}$ defines a network in the plane. We denote by $V$ the number of vertices of this network (i.e. points which correspond to the intersection of two lines) and we denote by $E$ the number of edges of the network (i.e. segments of one of the $\Lambda_{j}$ joining two distinct adjacent vertices). A half line included in one of the $\Lambda_{j}$, starting from a vertex and containing no other vertex, will be called a ray. Observe that the number of rays is equal to twice the number of lines. The connected components of

$$
\mathbf{R}^{2}-\left(\Lambda_{1} \cup \ldots \cup \Lambda_{L}\right)
$$

will be called domains and will be denoted by $\Omega_{1}, \ldots, \Omega_{D}$. We will see in Lemma 3.1, that it is possible to color the different domains defined by the lines $\Lambda_{1}, \ldots, \Lambda_{L}$, using only two colors (which will be denoted by +1 and -1 ) in such a way that two adjacent domains have different colors. The color of the domain $\Omega_{j}$ will be denoted by $\iota_{j} \in\{ \pm 1\}$. Observe that there are only two different ways to color the domains $\Omega_{1}, \ldots, \Omega_{D}$.

To state our result, it is convenient to introduce a parameter $\epsilon>0$ and look for bounded solutions of

$$
\epsilon^{2} \Delta u+u-u^{3}=0
$$

which are defined in $\mathbf{R}^{2}$. Observe that, since we are looking for entire solutions, studying (1.1) or (2.1) is completely equivalent and in fact one can go back and forth from a solution of the former equation to a solution of the latter using a simple scaling

$$
v(\cdot)=u(\epsilon \cdot)
$$


We will try to be consistent with the notations and $u, \tilde{u}, u_{j}, \ldots$ will denote solutions of (2.1) while $v, \bar{v}, v_{s}, v_{j}, \ldots$ will denote solutions of (1.1).

This being understood, we have the :

Theorem 2.1. Assume that $\Lambda_{1}, \ldots, \Lambda_{L}$ is an admissible set of affine lines. Then, there exist a sequence $\left(\epsilon_{n}\right)_{n \geq 0}$ tending to 0 and a sequence $\left(u_{n}\right)_{n \geq 0}$ of solutions of (2.1) with $\epsilon=\epsilon_{n}$ which, for $j=1, \ldots, D$, converges, uniformly on compacts of $\Omega_{j}$, to the constant function equal to $\iota_{j}$ as $n$ tends to infinity. Moreover,

(i) the nodal set of $u_{n}$ converges (in Hausdorff distance) to $\Lambda_{1} \cup \ldots \cup \Lambda_{L}$ on compacts of $\mathbf{R}^{2}$;

(ii) the ends of $u_{n}$ converge to the affine lines $\Lambda_{1}, \ldots, \Lambda_{L}$,

as $n$ tends to infinity.

Let us comment on the last statement of the result. By definition, we will say that a sequence of affine lines, defined by a sequence of equations of the form $\mathrm{a}_{n} \cdot \mathrm{x}+b_{n}=0$ where $\mathrm{a}_{n} \in \mathbf{R}^{2}$ is a unit vector and $b_{n} \in \mathbf{R}$, converges to the affine line defined by the equation $\mathbf{a} \cdot \mathbf{x}+b=0$, if the sequence $\left(\mathrm{a}_{n}, b_{n}\right)_{n \geq 0}$ converges to $(\mathrm{a}, b)$.

For a generic choice of the set of admissible lines $\Lambda_{1}, \ldots, \Lambda_{L}$ our result can be made more precise. Indeed, given a subset $\Theta \subset\left(0, \frac{\pi}{2}\right]$ we will say that the affine lines $\Lambda_{1}, \ldots, \Lambda_{L}$ are in general position with respect to $\Theta$ if the angle (the one in $\left.\left(0, \frac{\pi}{2}\right]\right)$ between any two of these affine lines does not belong to $\Theta$. We then have the :

Theorem 2.2. There is a finite set $\Theta \subset\left(0, \frac{\pi}{2}\right]$ (possibly empty) such that, if $\Lambda_{1}, \ldots, \Lambda_{L}$ is an admissible set of affine lines which are in general position with respect to $\Theta$, then for all $\epsilon>0$ close enough to 0 , there exists $u_{\epsilon}$, solution of (2.1) which, for $j=1, \ldots, D$, converges, uniformly on compacts of $\Omega_{j}$, to the constant function equal to $\iota_{j}$ as $\epsilon$ tends to 0 . Moreover, for each $j=1, \ldots, L$, there is one end of $u_{\epsilon}$ which is included in $\Lambda_{j}$ and

(i) the nodal set of $u_{\epsilon}$ converges (in Hausdorff distance) to $\Lambda_{1} \cup \ldots \cup \Lambda_{L}$ on compacts of $\mathbf{R}^{2}$;

(ii) the ends of $u_{\epsilon}$ converge to the affine lines $\Lambda_{1}, \ldots, \Lambda_{L}$,

as $\epsilon$ tends to 0 .

In other words, for a generic choice of the affine lines $\Lambda_{1}, \ldots, \Lambda_{L}$, one can find solutions of (2.1) which have half of their ends prescribed in the sense that, for each $j=1, \ldots, L$, they have one end included in $\Lambda_{j}$. Since the space of $L$ affine lines is $2 L$-dimensional and since the formal dimension of the space of solutions of (2.1) is $2 L$-dimensional, this result can also be interpreted by saying that, for $\epsilon$ close to 0 , the set of solutions of (2.1) can be locally parameterized by the affine lines $\Lambda_{1}, \ldots, \Lambda_{L}$.

\section{Construction of the approximate solution}

The main ingredient in the construction is a set of admissible affine lines $\Lambda_{1}, \ldots, \Lambda_{L}$. We first prove that it is possible to color the domains $\Omega_{1}, \ldots, \Omega_{D}$ defined by $\Lambda_{1}, \ldots, \Lambda_{L}$, using only two colors (which will be denoted by +1 and -1 ) in such a way that two adjacent domains have different colors.

Lemma 3.1. Assume that $L \geq 1$, then two colors suffice to distinguish the different domains of $\mathbf{R}^{2}-\left(\Lambda_{1} \cup \ldots \cup \Lambda_{L}\right)$. 
Proof. The result certainly holds when $L=1$ since one can assign the color +1 to one of the half planes of $\mathbf{R}-\Lambda_{1}$ and the color -1 to the other half plane. Assume that the result is true for the domains of $\mathbf{R}^{2}-\left(\Lambda_{1} \cup \ldots \cup \Lambda_{L}\right)$, namely, that it is possible to color them in such a way that two adjacent domains have different colors. Let us consider the domains of $\mathbf{R}^{2}-\left(\Lambda_{1} \cup \ldots \cup \Lambda_{L+1}\right)$ and assign to them the color they inherit from the fact that we were able to color the domains of $\mathbf{R}^{2}-\left(\Lambda_{1} \cup \ldots \cup \Lambda_{L}\right)$.

The line $\Lambda_{L+1}$ divides the plane into two half planes. Consider the domains included in one of the half plane and change their color systematically, i.e., change +1 into -1 and vice-versa. We have then colored the domains of $\mathbf{R}^{2}-\left(\Lambda_{1} \cup \ldots \cup\right.$ $\left.\Lambda_{L+1}\right)$ in such a way that two adjacent domains do not have the same color.

It is clear that there are two different ways to color the domains, since given a choice of assignment of \pm 1 to the components of $\mathbf{R}^{2}-\left(\Lambda_{1} \cup \ldots \cup \Lambda_{L}\right)$, we can change the sign assigned to each component and get a second coloring of the domains.

The second ingredient in the proof of Theorem 2.1 is the space $\mathcal{M}_{4}$ of 4-ended solutions of (1.1). Recall that a 4-ended solution of (1.1) is a solution whose nodal set is, outside a large ball, the union of four curves, each of which is asymptotic to a half affine line and furthermore, along each end, the solution is asymptotic to a heteroclinic solution having this affine line as a nodal set (a slightly different but equivalent definition was given by Gui [6]). The key fact about 4-ended solutions is that, after a rigid motion, they are even and, in the first quadrant

$$
Q^{\llcorner}:=\left\{(x, y) \in \mathbf{R}^{2}: x>0, y>0\right\},
$$

they are monotone functions of the $x$ and the $y$ variables(see [6] for details and [7] for related results concerning traveling wave solutions of the Allen-Cahn equation). More precisely, if $v \in \mathcal{M}_{4}$ is a 4-ended solution, there exists a rigid motion $g$ of $\mathbf{R}^{2}$ such that the function $\bar{v}:=v \circ g$ satisfies

$$
\bar{v}(x, y)=\bar{v}(-x, y)=\bar{v}(x,-y)=\bar{v}(-x,-y),
$$

in $\mathbf{R}^{2}$ and

$$
\partial_{x} \bar{v}<0 \quad \text { and } \quad \partial_{y} \bar{v}>0 \text {, }
$$

in $Q^{\llcorner}$.

The subfamily of $\mathcal{M}_{4}$ which consists of functions satisfying the above symmetries will be denoted by $\mathcal{M}_{4}^{\text {even }}$. Because of even symmetry and the monotonicity property, the nodal set of a solution $v \in \mathcal{M}_{4}^{\text {even }}$, restricted to $Q^{\llcorner}$, consists of a single curve, which is asymptotic to the half of an affine line $\Lambda$. We denote by $\theta \in\left(0, \frac{\pi}{2}\right)$, the angle between the $x$-axis and $\Lambda$ and we define the angle map by

$$
\begin{aligned}
\mathcal{A}: \mathcal{M}_{4}^{\text {even }} & \rightarrow\left(-\frac{\pi}{4}, \frac{\pi}{4}\right) \\
v & \mapsto \frac{\pi}{4}-\theta .
\end{aligned}
$$

For the saddle solution constructed in [2], we have $\mathcal{A}(v)=0$, while, for the solutions with almost parallel ends which were constructed in [4] we have $\mathcal{A}(v) \sim \pm \frac{\pi}{4}$. In [9] and [10], it is proven that $\mathcal{M}_{4}^{\text {even }}$ is diffeomorphic to $\mathbf{R}$ and also that the angle map is surjective. In particular, for each $\alpha \in\left(-\frac{\pi}{4}, \frac{\pi}{4}\right)$ there exists (at least) one element $v \in \mathcal{M}_{4}^{\text {even }}$ such that $\mathcal{A}(v)=\alpha$.

Remark 3.1. It is very likely that $\mathcal{A}$ is in fact one-to-one but this is still an open problem. 

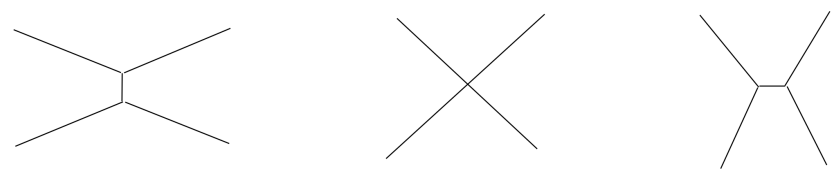

FIGURE 1. Schematic picture corresponding to a 4-ended solution with axis of symmetry the $x$-axis and $y$-axis. The picture on the left corresponds to the case where $\mathcal{A}(v)>0$, the picture in the middle corresponds to the saddle solution, namely to the case where $\mathcal{A}(v)=0$ and the picture on the right corresponds to the case where $\mathcal{A}(v)<0$.

Let us now turn to one of the most important step in this paper, namely the construction of the approximate solution. It will be obtained by gluing together 4-ended solutions at the vertices of the network defined by the straight lines $\Lambda_{1}, \ldots, \Lambda_{L}$. To begin with, let us describe more carefully the elements of $\mathcal{M}_{4}^{\text {even }}$. By definition, these solutions of (1.1) have two axes of symmetry (the $x$-axis and the $y$-axis) and, because of these symmetries, in order to describe the ends of a solution $v \in \mathcal{M}_{4}^{\text {even }}$, it is enough to describe the end of $v$ which is included in $Q^{\llcorner}$. Observe that the ends of $v$ are asymptotic to 4 half affine lines which do not necessarily meet at the same point. We refer to Figure 1 where we have drawn the half lines associated to the ends of elements in $\mathcal{M}_{4}^{\text {even }}$ according to the angle between the ends of the solution.

To construct the approximate solution starting from a set of admissible affine lines, we need to explain how to choose and insert 4-ended solutions at the vertices of the network defined by these lines. We follow the approach of Traizet [12]. We first rotate the set of affine lines $\Lambda_{1}, \ldots, \Lambda_{L}$ in such a way that their $y$ coordinates are strictly ordered. Hence, we assume from now on that vertices of the network associated to $\Lambda_{1}, \ldots, \Lambda_{L}$ are given by $\mathrm{x}_{1}, \ldots, \mathrm{x}_{V}$ and if $\mathrm{x}_{j}=\left(x_{j}, y_{j}\right)$, then

$$
y_{j+1}<y_{j}
$$

for $j=1, \ldots, V-1$. Thanks to Lemma 3.1, we can also assume that the different domains $\Omega_{j}$, for $j=1, \ldots, D$ associated to $\Lambda_{1}, \ldots, \Lambda_{L}$ have been assigned a color $\iota_{j} \in\{ \pm 1\}$. The construction of an approximate solution is now decomposed into three different steps.

Step 1. We choose the vertex with the largest $y$ coordinate, namely $\mathrm{x}_{1}$. Two of the affine lines $\Lambda_{1}, \ldots, \Lambda_{L}$ meet at $\mathrm{x}_{1}$, say $\Lambda_{j_{1}}$ and $\Lambda_{j_{1}^{\prime}}$. The angle between $\Lambda_{j_{1}}$ (resp. $\left.\Lambda_{j_{1}^{\prime}}\right)$ and the $x$-axis will be denoted by $\alpha_{1} \in\left[-\frac{\pi}{2}, \frac{\pi}{2}\right]\left(\right.$ resp. $\left.\alpha_{1}^{\prime} \in\left[-\frac{\pi}{2}, \frac{\pi}{2}\right]\right)$ and, without loss of generality, we can assume that $\alpha_{1}>\alpha_{1}^{\prime}$. We choose an element $v_{1} \in \mathcal{M}_{4}^{\text {even }}$ whose angle corresponds to $\alpha_{1}-\alpha_{1}^{\prime}$, namely

$$
\mathcal{A}\left(v_{1}\right)=\frac{\pi}{4}-\frac{\alpha_{1}-\alpha_{1}^{\prime}}{2} .
$$

Next, we define the model solution $u_{1}$ of (2.1) which is centered at $\mathrm{x}_{1}$ and whose ends are parallel to $\Lambda_{j_{1}}$ and $\Lambda_{j_{1}^{\prime}}$ by

$$
u_{1}(\mathrm{x}):=s_{1} v_{1}\left(\frac{\mathcal{R}_{\frac{\alpha_{1}+\alpha_{1}^{\prime}}{2}}\left(\mathrm{x}-\mathrm{x}_{1}\right)}{\epsilon}\right),
$$



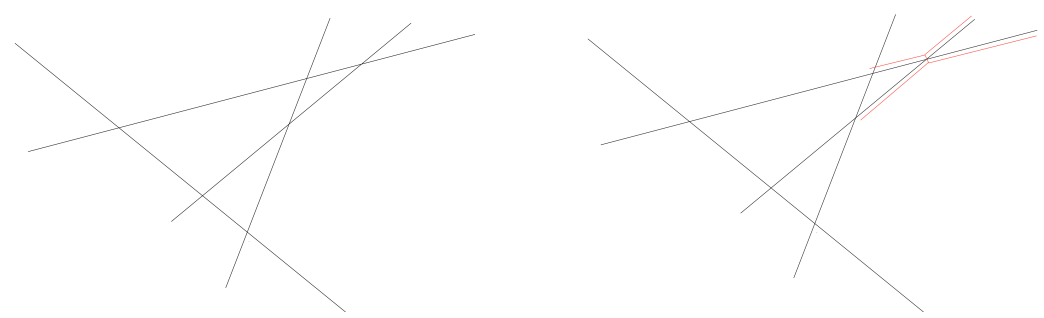

FIgURE 2. The initial configuration of 4 lines and Step 1. The model solution is drawn in red and centered at the vertex, its ends are parallel to the ends of the two lines meeting at the vertex.
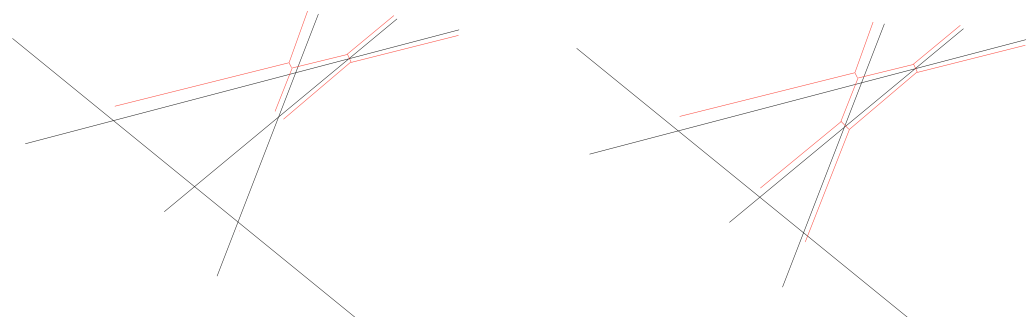

FiguRE 3. The first picture corresponds to Case 2 where the model solution has to be translated so that one of its ends coincides with one of the ends of the model solution already inserted at an earlier step. The second picture corresponds to Case 3 where the model solution has to be translated so that two of its ends coincide with the ends of two model solutions inserted at earlier steps.

where $\mathcal{R}_{\alpha}$ is the clockwise rotation of angle $\alpha$ in the plane and $s_{1} \in\{ \pm 1\}$. Observe, and this is important, that the ends of $u_{1}$ are not included in $\Lambda_{j_{1}}$ and $\Lambda_{j_{1}^{\prime}}$ but are simply parallel to these lines as illustrated in Figure 2. Also observe that the distance between the ends of $u_{1}$ and $\Lambda_{j_{1}} \cup \Lambda_{j_{1}^{\prime}}$ is bounded by a constant times $\epsilon$. Finally, $s_{1}$ is chosen so that the domains where $u_{1}$ is positive (negative) agree with the colors of the four domains which have a common boundary with both $\Lambda_{j_{1}}$ and $\Lambda_{j_{1}^{\prime}}$. We also define $\tilde{\mathrm{x}}_{1}:=\mathrm{x}_{1}$.

Step 2. We repeat the above construction inductively. Let us assume that we have already constructed a model solution at the vertices $\mathrm{x}_{1}, \ldots, \mathrm{x}_{k-1}$ and let us explain how to construct the model solution of (2.1) at $\mathrm{x}_{k}$.

Again, we consider the angles $\alpha_{k}>\alpha_{k}^{\prime} \in\left[-\frac{\pi}{2}, \frac{\pi}{2}\right]$ between the two lines $\Lambda_{j_{k}}$ and $\Lambda_{j_{k}^{\prime}}$ meeting at $\mathrm{x}_{k}$ and the $x$-axis, and we choose an element $v_{k} \in \mathcal{M}_{4}^{e v e n}$ such that

$$
\mathcal{A}\left(v_{k}\right)=\frac{\pi}{4}-\frac{\alpha_{k}-\alpha_{k}^{\prime}}{2} .
$$

As in the first step, we then use a rigid motion and scaling by $\epsilon$, to produce, starting from $v_{k}$, a solution $u_{k}$ of $(2.1)$ which is centered at $\mathrm{x}_{k}$, whose ends are parallel to the lines meeting at this vertex and we choose the sign which agrees with the coloring of the domains bounded by both $\Lambda_{j_{k}}$ and $\Lambda_{j_{k}^{\prime}}$.

Three cases have to be distinguished.

Case 1. The first case corresponds to the case where $\Lambda_{j_{k}}$ and $\Lambda_{j_{k}^{\prime}}$ do not contain any of the vertices $\mathrm{x}_{1}, \ldots, \mathrm{x}_{k-1}$, in which case we are done. We set 
$\tilde{\mathrm{x}}_{k}:=\mathrm{x}_{k}$ and choose

$$
u_{k}(\mathrm{x}):=s_{k} v_{k}\left(\frac{\mathcal{R}_{\frac{\alpha_{k}+\alpha_{k}^{\prime}}{2}}\left(\mathrm{x}-\tilde{\mathrm{x}}_{k}\right)}{\epsilon}\right)
$$

as the model solution at $\mathrm{x}_{k}$, where $s_{k} \in\{ \pm 1\}$.

Case 2. The second case corresponds to the case where one of the lines meeting at $\mathrm{x}_{k}$ contains some of the vertices $\mathrm{x}_{1}, \ldots, \mathrm{x}_{k-1}$ while the other line does not contain any of these vertices. Say for example that $\Lambda_{j_{k}}$ contains some of the vertices $\mathrm{x}_{1}, \ldots, \mathrm{x}_{k-1}$ while $\Lambda_{j_{k}^{\prime}}$ does not. Observe that, necessarily, these vertices are all contained in one of the two connected components of $\Lambda_{j_{k}}-\left\{\mathrm{x}_{k}\right\}$ (this is where we use the fact that the vertices are ordered according to their height and hence the $y$-coordinates of $\mathrm{x}_{1}, \ldots, \mathrm{x}_{k-1}$ are all larger than the $y$-coordinate of $\mathrm{x}_{k}$ ). Let us denote by $\mathrm{x}_{j}$ the vertex in the collection $\mathrm{x}_{1}, \ldots, \mathrm{x}_{k-1}$ which belongs to $\Lambda_{j_{k}}$ and which is the closest to $\mathrm{x}_{k}$.

We define $u_{k}$ by (3.2) as the model solution at $\mathrm{x}_{k}$, where $s_{k} \in\{ \pm 1\}$ and where $\tilde{x}_{k}$ is the unique point which belongs to the affine line passing through $\mathrm{x}_{k}$ and orthogonal to $\Lambda_{j_{k}}$, which is chosen so that the end of $u_{k}$, which is parallel to $\Lambda_{j_{k}}$ and points towards $\mathrm{x}_{j}$, coincides with the end of $u_{j}$, which is also parallel to $\Lambda_{j_{k}}$ and points towards $\mathrm{x}_{k}$. Observe that the center $\tilde{\mathbf{x}}_{k}$ of $u_{k}$ is at most at distance $\mathcal{O}(\epsilon)$ from $\mathbf{x}_{k}$. This is the configuration depicted in the first picture of Figure 3.

Case 3. The third case we need to consider corresponds to the case where both $\Lambda_{j_{k}}$ and $\Lambda_{j_{k}^{\prime}}$ contain some of the vertices $\mathrm{x}_{1}, \ldots, \mathrm{x}_{k-1}$. Since we have ordered the vertices according to their height, the vertices $\mathrm{x}_{1}, \ldots, \mathrm{x}_{k-1}$ belonging to $\Lambda_{j_{k}}$ and $\Lambda_{j_{k}^{\prime}}$ are included in one of the connected components of $\Lambda_{j_{k}}-\left\{\mathrm{x}_{k}\right\}$ and $\Lambda_{j_{k}^{\prime}}-\left\{\mathrm{x}_{k}\right\}$. Let us denote by $\mathrm{x}_{j}\left(\right.$ resp. $\left.\mathrm{x}_{j^{\prime}}\right)$ the vertex in the collection $\mathrm{x}_{1}, \ldots, \mathrm{x}_{k-1}$ which belongs to $\Lambda_{j_{k}}\left(\right.$ resp. $\left.\Lambda_{j_{k}^{\prime}}\right)$ and which is the closest to $\mathrm{x}_{k}$.

We define $u_{k}$ by (3.2) as the model solution at $\mathrm{x}_{k}$, where $s_{k} \in\{ \pm 1\}$ and where $\tilde{x}_{k}$ is the unique point chosen so that the end of $u_{k}$ which is parallel to $\Lambda_{j_{k}}$ and points towards $\mathrm{x}_{j}$, coincides with the end of $u_{j}$ which is also parallel to $\Lambda_{j_{k}}$ and points towards $\mathrm{x}_{k}$ and we also require that the end of $u_{k}$ which is parallel to $\Lambda_{j_{k}^{\prime}}$ and points towards $\mathrm{x}_{j}^{\prime}$ coincides with the end of $u_{j^{\prime}}$ which is also parallel to $\Lambda_{j_{k}^{\prime}}$ and points towards $\mathrm{x}_{k}$. Observe that the center $\tilde{\mathrm{x}}_{k}$ of $u_{k}$ is at a distance $\mathcal{O}(\epsilon)$ from $\mathrm{x}_{k}$. This is the configuration depicted in the second picture of Figure 3.

In each case, $s_{k} \in\{ \pm 1\}$ is chosen so that the domains where $u_{k}$ is positive (negative) agree with the colors of the four domains which have a common boundary with both $\Lambda_{j_{k}}$ and $\Lambda_{j_{k}^{\prime}}$.

Step 3. To complete the construction of the approximate solution, we use appropriate cutoff functions to glue together the model solutions $u_{1}, \ldots, u_{V}$ at the different vertices. To this aim, we need to define a partition of unity. For each $j=1, \ldots, V$, we remove from the network defined by the lines $\Lambda_{1}, \ldots, \Lambda_{L}$, the edges and rays starting at $\mathrm{x}_{j}$. Call $T_{j}$ this network and $S_{j}$ the set of rays starting at $\mathrm{x}_{j}$ (so $S_{j}$ contains two, one or no ray and in the latter case $S_{j}$ deduces to $\left\{\mathrm{x}_{j}\right\}$ ). We then consider $U_{j}$ to be the open connected component of $\mathbf{R}^{2}-T_{j}$, which contains 


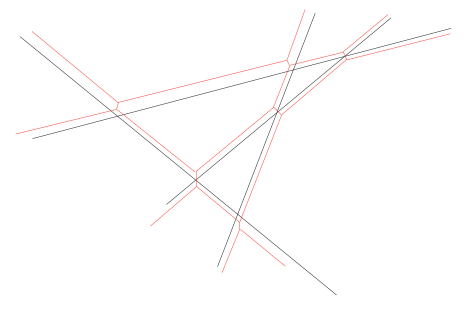

FIGURE 4. The final configuration with all the model solutions inserted near the vertices of the network defined by the 4 original lines.

$\mathrm{x}_{j}$. Then

$$
O_{j}:=\left\{\mathrm{x}: \operatorname{dist}\left(\mathrm{x}, S_{j}\right) \leq \operatorname{dist}\left(\mathrm{x}, \mathbf{R}^{2}-U_{j}\right)-\eta_{j}\right\},
$$

where the $\eta_{j}>0$ are chosen in such a way that $\operatorname{dist}\left(O_{j}, O_{k}\right)>0$ for $j \neq k$. We also consider an open cover of $\mathbf{R}^{2}$ given by the sets:

$$
\left\{\mathrm{x}: \operatorname{dist}\left(\mathrm{x}, S_{j}\right) \leq \operatorname{dist}\left(\mathrm{x}, \mathbf{R}^{2}-U_{j}\right)+\eta_{j}\right\}
$$

and assume that we are given a partition of unity $\chi_{1}, \ldots, \chi_{V}$ subordinate to it and such that

$$
\sum_{j=1}^{V} \chi_{j} \equiv 1,
$$

and

$$
\chi_{j} \equiv\left\{\begin{array}{lll}
1 & \text { in } & O_{j}, \\
0 & \text { in } & \bigcup_{k \neq j} O_{k} .
\end{array}\right.
$$

We further assume that

$$
\left\|\chi_{j}\right\|_{\mathcal{C}^{\infty}\left(\mathbf{R}^{2}\right)} \leq C .
$$

Given a function $f$ defined in $\mathbf{R}^{2}$, it will be convenient to adopt the notation

$$
K_{j}^{*} f(\mathrm{x}):=f\left(\frac{\mathcal{R}_{\alpha_{j}+\alpha_{j}^{\prime}}\left(\mathrm{x}-\tilde{\mathrm{x}}_{j}\right)}{\epsilon}\right),
$$

so that the model solutions defined above are given by

$$
u_{j}=s_{j} K_{j}^{*} v_{j}
$$

where we recall that $s_{j} \in\{ \pm 1\}$ and $v_{j} \in \mathcal{M}_{4}^{e v e n}$. The approximate solution $\tilde{u}_{\epsilon}$ is defined by

$$
\tilde{u}_{\epsilon}:=\sum_{j=1}^{V} \chi_{j} u_{j} .
$$

Observe that $\tilde{u}_{\epsilon}$ depends on $\epsilon$ since the model solutions $u_{1}, \ldots, u_{V}$ do. Also, as $\epsilon$ tends to 0 , the sequence $\tilde{u}_{\epsilon}$ satisfies the statement of Theorem 2.1, apart from being a solution of (2.1). Finally, $\tilde{u}_{\epsilon}$ is an approximate solution of (2.1) in the sense that

$$
\left\|\epsilon^{2} \Delta \tilde{u}_{\epsilon}+\tilde{u}_{\epsilon}-\tilde{u}_{\epsilon}^{3}\right\|_{L^{\infty}\left(\mathbf{R}^{2}\right)} \leq C e^{-\frac{c}{\epsilon}}
$$

for some constants $c, C>0$, as can be checked using the fact that elements of $\mathcal{M}_{4}^{\text {even }}$ converge to the heteroclinic solutions exponentially fast along their ends (c.f. [3] and [9]). The constants $c$ and $C$ do not depend $\epsilon$ but do depend on the 
$v_{j}, j=1, \ldots, V$, used to construct $\tilde{u}_{\epsilon}$, since they are related to the exponential rate at which the functions $v_{j}$ approach the heteroclinic solutions along their ends.

\section{Comments on the Construction of the approximate solution}

The following Lemma holds :

Lemma 4.1. Assume that $L \geq 2$, then the following formulae holds :

$$
V=\frac{L(L-1)}{2}, \quad E=L(L-2) \quad \text { and } \quad D=1+\frac{L(L+1)}{2} .
$$

Proof. The formulae hold when $L=2$, since in this case $V=1, E=0$ and $D=4$. Assume that the result is true for the lines $\Lambda_{1}, \ldots, \Lambda_{L}$. Addition of one line $\Lambda_{L+1}$ increases the number of lines by 1 , the number of vertices by $L$ since $\Lambda_{L+1}$ has one intersection point with each $\Lambda_{j}$ for $j=1, \ldots, L$ and also increases the number of edges by $2 L-1$ since we have created $L$ new edges included in the lines $\Lambda_{1}, \ldots, \Lambda_{L}$ and also $L-1$ new edges included in $\Lambda_{L+1}$. Finally, using similar arguments, one checks that it increases the number of domains by $L+1$. Using this, one proves the formula by induction on $L$.

Observe that

$$
2 L=4 V-2 E .
$$

This can be interpreted as follows : $2 L$ is the expected dimension of the space of solutions for the Allen-Cahn equation which have $2 L$ ends. For each vertex there is a 4 dimensional family of model solutions which can be used for the approximate solution (namely solutions with 4 ends up to rotation and translations). Hence the number of degrees of freedom in the construction is $4 \mathrm{~V}$. Because the ends of the different model solutions used at each vertex should match along the edges, there are 2 constrains for each edge and hence a total of $2 E$ equations to solve. So the formula just states that the expected dimension of the space of solutions is equal to the number of degrees of freedom in the construction, minus the number of equations we need to solve for the construction to be successful.

Our result parallels Traizet's construction of Scherk towers [12] and in fact this is where we borrowed the idea of the construction in the present paper. Let us recall that a Scherk surface is an embedded minimal surface which is singly periodic and has 4 ends asymptotic to 4 vertical half planes. Once the vertical period of the surface is fixed, these surfaces come in a one parameter family parameterized by the angle between two consecutive ends of the surface. Starting from an initial configurations of vertical planes, Traizet was able to prove the existence of a family of minimal surfaces which desingularize this collection of planes using Scherk surfaces. In this way, he obtained embedded minimal surfaces in $\mathbf{R}^{3}$ which are singly periodic and asymptotic at infinity to a finite set of half planes, each of which is orthogonal to the horizontal plane. The correspondence between Traizet's construction and our construction is simply that the collection of lines in the plane replaces the collection of vertical planes in Traizet's construction and 4-ended solutions of (1.1) replace the family of Scherk surfaces in the context of minimal surfaces.

Similar ideas have then been used many times in differential geometry. Indeed our construction in the present paper as well as Traizet's construction fall into the category of what are now referred to as end-to-end constructions. In these 
constructions, one starts with a finite number of solutions of the problem which have the properties to have ends and one tries to produce new solutions by connecting together the different summands along their ends. This for example has been exploited by Ratzkin [11] and Jleli and Pacard [8] in the context of constant mean curvature surfaces.

\section{THE LINEARIZED OPERATOR ABOUT 4-ENDED SOLUTION}

In this section, we recall some basic facts about the linearized operator about a 4-ended solution of the Allen-Cahn equation. The results of this section can be found in [3] and [9]. We assume that $v \in \mathcal{M}_{4}^{\text {even }}$ is given and we define the linearized operator about $v$ by

$$
L_{v}:=\Delta+1-3 v^{2}
$$

The following result is proven in [9].

Theorem 5.1. Assume that $L_{v} \phi=0$ and also that $\phi \in e^{\delta \sqrt{1+|\mathrm{x}|^{2}}} L^{2}\left(\mathbf{R}^{2}\right)$ for some $\delta<0$. Then $\phi=0$.

This result states that the operator $L_{v}$ has no element in its kernel that decays exponentially fast to 0 at infinity. It is clear that the kernel of $L_{v}$ contains functions which are bounded and indeed, $\partial_{x} v$ and $\partial_{y} v$ are bounded functions which clearly belong to the kernel of $L_{v}$. This is a consequence of the invariance of our problem under the action of the elements of the group of translations. Similarly, $\left(y \partial_{x}-\right.$ $\left.x \partial_{y}\right) v$ also belongs to the kernel of $L_{v}$ and this reflects the invariance of our problem under the action of the elements of the group of rotations in the plane. Observe that this last function is not bounded but grows linearly at infinity.

Let us recall a few results from [3]. Let $\bar{\chi}_{\llcorner}, \bar{\chi}_{\lrcorner}, \bar{\chi}_{\urcorner}, \bar{\chi}_{\ulcorner}$be a smooth partition of unity of $\mathbf{R}^{2}$ subordinate to the four quadrants in the plane defined by the $x$-axis and the $y$-axis. We assume that

$$
\bar{\chi}_{\llcorner} \equiv\left\{\begin{array}{lll}
1 & \text { when } & x>1 \quad \text { and } y>1, \\
0 & \text { when } & x<-1 \text { or } y<-1,
\end{array}\right.
$$

and, without loss of generality, we can assume that $\bar{\chi}\lrcorner$ is obtained from $\bar{\chi}_{\llcorner}$by a symmetry with respect to the $y$-axis, $\bar{\chi}_{\ulcorner}$is obtained from $\bar{\chi}_{\llcorner}$by a symmetry with respect to the $x$-axis and $\bar{\chi}_{\urcorner}$is obtained from $\bar{\chi}_{\llcorner}$by a symmetry with respect to the origin. Finally, we assume that

$$
\left\|\bar{\chi}_{\llcorner}\right\|_{\mathcal{C}^{\infty}\left(\mathbf{R}^{2}\right)} \leq C
$$

We define

$$
v_{\llcorner}:=\bar{\chi}_{\llcorner} v,
$$

with similar definitions for $v_{\lrcorner}, v_{\urcorner}$and $v\ulcorner$.

The deficiency space $\mathfrak{D}_{v}$ associated to $v \in \mathcal{M}_{4}^{\text {even }}$ is the 8-dimensional vector space defined by

$$
\mathfrak{D}_{v}:=\operatorname{Span}\left\{\partial_{x} v_{\bullet},\left(x \partial_{y}-y \partial_{x}\right) v_{\bullet}: \bullet=\llcorner,\lrcorner,\right\urcorner,\ulcorner\} .
$$

To proceed, we assume that the equation of the end of $v$ which is included in $Q^{\llcorner}$ is given by

$$
\mathrm{a} \cdot \mathrm{x}+b=0,
$$


where a $\in \mathbf{R}^{2}$ is a unit vector which points in the upper half space and where $b \in \mathbf{R}$. Given $\tau, \delta<0$, we define a weight function

$$
\Gamma_{\tau, \delta}(\mathrm{x}):=\sum_{\bullet=\llcorner,\lrcorner,\urcorner,\ulcorner} \bar{\chi}_{\bullet}(\mathrm{x}) e^{\tau \mathrm{x}_{\bullet} \cdot \mathrm{a}^{\perp}}\left(\cosh \left(\mathrm{a} \cdot \mathrm{x}_{\bullet}+b\right)\right)^{\delta},
$$

where $\mathrm{a}^{\perp}$ is orthogonal to a and is interior to the quadrant $Q^{\llcorner}$, and where we have set

$$
\mathrm{x}_{\llcorner}:=(x, y), \quad \mathrm{x}_{\lrcorner}:=(-x, y), \quad \mathrm{x}_{\urcorner}:=(-x,-y) \quad \text { and } \quad \mathrm{x} r:=(x,-y),
$$

for $\mathrm{x}=(x, y)$. By construction, $\tau$ is the rate of decay along the end of $v$ and $\delta$ is the rate of decay in the direction orthogonal to the end of $v$. Note that the weight function depends on the asymptotic behavior of the four-end solution $v$. We will not make this dependence explicit in the notation unless necessary.

With this definition in mind, we define the weighted Lebesgue space

$$
L_{\tau, \delta}^{2}\left(\mathbf{R}^{2}\right):=\Gamma_{\tau, \delta} L^{2}\left(\mathbf{R}^{2}\right),
$$

and the weighted Sobolev space

$$
W_{\tau, \delta}^{2,2}\left(\mathbf{R}^{2}\right):=\Gamma_{\tau, \delta} W^{2,2}\left(\mathbf{R}^{2}\right) .
$$

It is important to notice that, even though we do not make this apparent in the notation, these function spaces do depend on the function $v \in \mathcal{M}_{4}^{\text {even }}$ since they depend on the definition of the ends of $v$. For later use, it will be important to make this dependance explicit in the notation and in this case we will write $L_{v, \tau, \delta}^{2}\left(\mathbf{R}^{2}\right)$ and $W_{v, \tau, \delta}^{2,2}\left(\mathbf{R}^{2}\right)$ instead of $L_{\tau, \delta}^{2}\left(\mathbf{R}^{2}\right)$ and $W_{\tau, \delta}^{2,2}\left(\mathbf{R}^{2}\right)$.

We now recall some facts about the moduli space theory developed in [3] and [9]. For example, we have the :

Proposition 5.1. For all $\tau, \delta<0$ close enough to 0 , the operator

$$
\begin{aligned}
\mathcal{L}_{v}: W_{\tau, \delta}^{2,2}\left(\mathbf{R}^{2}\right) \oplus \mathfrak{D}_{v} & \longrightarrow L_{\tau, \delta}^{2}\left(\mathbf{R}^{2}\right) \\
\phi & \longmapsto L_{v} \phi,
\end{aligned}
$$

is surjective and has a 4-dimensional kernel.

We now assume that $\tau, \delta<0$ are fixed close enough to 0 so that the above result holds. Clearly $\partial_{x} v, \partial_{y} v$ and $\left(x \partial_{y}-y \partial_{x}\right) v$ belong to the kernel of $\mathcal{L}_{v}$ and each of these has a decomposition in $W_{\tau, \delta}^{2,2}\left(\mathbf{R}^{2}\right) \oplus \mathfrak{D}_{v}$. Obviously

$$
\partial_{x} v-\sum_{\bullet=\llcorner,\lrcorner,\urcorner,\ulcorner} \partial_{x} v_{\bullet} \in W_{\tau, \delta}^{2,2}\left(\mathbf{R}^{2}\right),
$$

and we also have

$$
\partial_{y} v-\cot \theta\left(\sum_{\bullet=\lrcorner,\ulcorner} \partial_{x} v_{\bullet}-\sum_{\bullet=\llcorner,\urcorner} \partial_{x} v_{\bullet}\right) \in W_{\tau, \delta}^{2,2}\left(\mathbf{R}^{2}\right),
$$

where $\theta$ denotes the angle between $x$-axis and the end of $v$ in $Q^{\llcorner}$. The interested reader will find a similar decomposition for $\left(x \partial_{y}-y \partial_{x}\right) v$.

Remark 5.1. Thanks to (5.4), one can check that Proposition 5.1 still holds if, in the definition of $\mathfrak{D}_{v}$, one replaces $\partial_{x} v_{\bullet}$ by $\partial_{y} v_{\bullet}$ or by any (non zero) linear combination of $\partial_{x} v_{\bullet}$ and $\partial_{y} v_{\bullet}$. 
To proceed with the analysis of the kernel of $\mathcal{L}_{v}$, recall that it follows from the results in [3], [9] and [10], that $\mathcal{M}_{4}^{\text {even }}$ is a smooth one dimensional manifold diffeomorphic to $\mathbf{R}$. Moreover, if $s \mapsto v_{s}$ is a regular parametrization of $\mathcal{M}_{4}^{\text {even, }}$, then

$$
\phi_{s}:=\partial_{s} v_{s}
$$

belongs to the kernel of $\mathcal{L}_{v_{s}}$ and the question is to understand the decomposition of $\phi_{s}$ in the space $W_{\tau, \delta}^{2,2}\left(\mathbf{R}^{2}\right) \oplus \mathfrak{D}_{v_{s}}$. Here $\tau, \delta<0$ can be chosen close enough to 0 , independently of $s$ in a given compact of $\mathbf{R}$.

Observe that $\phi_{s}$ is symmetric with respect to both the $x$-axis and the $y$-axis since elements of $\mathcal{M}_{4}^{\text {even }}$ also are. Hence, to understand the decomposition of $\phi_{s}$, it is enough to restrict our attention to $Q^{\llcorner}$where we can write

$$
\phi_{s}-\left(\lambda_{s}\left(y \partial_{x}-x \partial_{y}\right) v_{s}+\mu_{s} \partial_{x} v_{s}\right) \in \Gamma_{\tau, \delta} L^{2}\left(Q^{\llcorner}\right),
$$

for some $\lambda_{s}, \mu_{s} \in \mathbf{R}$. By assumption $\phi_{s} \neq 0$ and, thanks to Theorem 5.1 we conclude that $\left(\lambda_{s}, \mu_{s}\right) \neq(0,0)$.

If $\lambda_{s} \neq 0$, then $\phi_{s}$ is not bounded (and in fact grows linearly). From a geometric point of view, this also implies that, close to $v_{s}$, the moduli space $\mathcal{M}_{4}^{\text {even }}$ can be parameterized by the angle function and in fact

$$
\lambda_{s}=\partial_{s} \theta_{s}
$$

where $s \mapsto \theta_{s}$ is defined by the identity

$$
\mathcal{A}\left(v_{s}\right)=\frac{\pi}{4}-\theta_{s}
$$

We have already mentioned that the mapping $\mathcal{A}$ defined in (3.1) is onto and this implies that, as the parameter $s$ varies, the value of $s \mapsto \theta_{s}$ varies from 0 to $\frac{\pi}{2}$. Intuitively, this means that, although it may happen that $\lambda_{s}=0$, there should exist a large set of parameters $s$ for which $\lambda_{s} \neq 0$. It is quite natural to conjecture that $\lambda_{s} \neq 0$ for all $s \in \mathbf{R}$, but unfortunately this result is beyond our reach and we show instead :

Lemma 5.1. There exists a finite set $\mathcal{S} \subset \mathbf{R}$ (possibly empty) such that, for all $s \in \mathbf{R}-\mathcal{S}, \lambda_{s} \neq 0$.

Proof. It follows from the construction in [4] that $\lambda_{s} \neq 0$ whenever $|s|$ is large. Now the key observation is that $\mathcal{M}_{4}^{\text {even }}$ is a real analytic manifold (this follows from the results in [3] and the analytic version of the implicit function theorem in the proof of Theorem 2.2 in [3]). This implies that $\lambda_{s}=0$ for at most finitely many values of $s \in \mathbf{R}$ and this completes the proof of the lemma.

Definition 5.1. We will say that $v \in \mathcal{M}_{4}^{\text {even }}$ is regular if $v=v_{s}$ for some $s \in \mathbf{R}-\mathcal{S}$.

This result has an important consequence which we now describe. Assume that the end of $v$ which is included in the upper quadrant $Q^{\llcorner}$is defined by the equation

$$
\mathrm{a} \cdot \mathrm{x}+b=0
$$

where a is a unit vector which points towards the interior of the upper half space. Then, along this end, the elements in $\mathfrak{D}_{v}$ are asymptotic to a linear combination of the functions

$$
\mathbf{t}(\mathrm{x}):=\operatorname{sech}^{2}\left(\frac{\mathrm{a} \cdot \mathrm{x}+b}{\sqrt{2}}\right)
$$


and

$$
\mathbf{r}(\mathrm{x}):=\operatorname{sech}^{2}\left(\frac{\mathrm{a} \cdot \mathrm{x}+b}{\sqrt{2}}\right) \mathrm{x} \cdot \mathrm{a}^{\perp} .
$$

In particular, given $\phi \in \operatorname{Ker} \mathcal{L}_{v}$, one can find $\kappa_{\mathbf{t}}^{\bullet}, \kappa_{\mathbf{r}}^{\bullet} \in \mathbf{R}$, for $\left.\bullet=\llcorner\lrcorner,\right\urcorner,\ulcorner$ such that

$$
\phi-\sum_{\bullet=L,\lrcorner,\urcorner,\ulcorner} \bar{\chi}_{\bullet}(\mathrm{x})\left(\kappa_{\mathbf{t}}^{\bullet} \mathbf{t}\left(\mathrm{x}_{\bullet}\right)+\kappa_{\mathbf{r}}^{\bullet} \mathbf{r}\left(\mathrm{x}_{\bullet}\right)\right) \in e^{-c \sqrt{1+|\mathrm{x}|^{2}}} L^{2}\left(\mathbf{R}^{2}\right),
$$

for some $c>0$.

The fact that $v \in \mathcal{M}_{4}^{\text {even }}$ is regular translates into the following:

Proposition 5.2. Assume that $v \in \mathcal{M}_{4}^{\text {even }}$ is regular in the sense of Definition 5.1. Then, one can find an element in $\operatorname{Ker} \mathcal{L}_{v}$ whose coefficients $\kappa_{\mathbf{t}}^{\llcorner}, \kappa_{\mathbf{r}}^{\llcorner}, \kappa_{\mathbf{t}}^{\lrcorner}, \kappa_{\mathbf{r}}^{\lrcorner}$are prescribed.

Proof. Adding to $\phi$ a linear combination of $\partial_{x} v$ and $\partial_{y} v$ allows one to prescribe the coefficients $\kappa_{\mathbf{t}}^{\llcorner}$and $\kappa_{\mathbf{t}}^{\lrcorner}$. Thanks to Lemma 5.1, adding to $\phi$ a linear combination of $\left(x \partial_{y}-y \partial_{x}\right) v$ and $\partial_{s} v_{s}$ for $s \in \mathbf{R}$ chosen so that $v=v_{s}$, allows one to prescribe the coefficients $\kappa_{\mathbf{r}}^{\llcorner}$and $\kappa_{\mathbf{r}}^{\lrcorner}$.

A similar result holds for the coefficients $\kappa_{\mathbf{t}}, \kappa_{\mathbf{r}}, \kappa_{\mathbf{t}}, \kappa_{\mathbf{r}}$ and in fact, one can prescribe the coefficients in the decomposition (5.5) of the elements of $\operatorname{Ker} \mathcal{L}_{v}$ in any of the half planes $\left\{(x, y) \in \mathbf{R}^{2}: \pm x>0\right\}$ or $\left\{(x, y) \in \mathbf{R}^{2}: \pm y>0\right\}$. However, it is not possible to prescribe the asymptotic behavior of an element of $\operatorname{Ker} \mathcal{L}_{v}$ in the upper right and the lower left quadrants. For example, it is not possible to find an element of $\operatorname{Ker} \mathcal{L}_{v}$ with the coefficients $\kappa_{\mathbf{t}}^{\llcorner}, \kappa_{\mathbf{r}}^{\llcorner}, \kappa_{\mathbf{t}}^{\urcorner}, \kappa_{\mathbf{r}}^{\urcorner}$prescribed.

\section{Gluing parametrices tOGether}

In this section, we would like analyze the mapping property of the linearized operator of the Allen-Cahn equation around the approximate solution. To begin with, let us define the function spaces we will work with. The definition will make use of the partition of unity already used in the construction of the approximate solution $\tilde{u}_{\epsilon}$. In a nutshell, the idea is to patch together the weighted Lebesgue and Sobolev spaces we have defined in the last section.

Given a function $f$ in $\mathbf{R}^{2}$ we have defined (see (3.3)) the operators:

$$
K_{j}^{*} f(\mathrm{x}):=f\left(\frac{R_{\alpha_{j}+\alpha_{j}^{\prime}}\left(\mathrm{x}-\tilde{\mathrm{x}}_{j}\right)}{\epsilon}\right), \quad j=1, \ldots, V .
$$

It is convenient to introduce the inverses of the operators $K_{j}^{*}$ :

$$
H_{j}^{*} f(\mathrm{x}):=f\left(\tilde{\mathrm{x}}_{j}+\epsilon R_{-\frac{\alpha_{j}+\alpha_{j}^{\prime}}{2}} \mathrm{x}\right) .
$$

Now, we will define the weighted spaces we will work with. Let us recall the definition of the approximate solution $\tilde{u}_{\epsilon}$ in (3.4). In the set $\operatorname{supp} \chi_{j}$ we have $\tilde{u}_{\epsilon} \approx s_{j} K_{j}^{*} v_{j}$, where $v_{j}$ is a 4-ended solution. We associate with this $v_{j}$ a weighted space $L_{v_{j}, \tau_{j}, \delta_{j}}^{2}\left(\mathbf{R}^{2}\right)$ with some $\tau_{j}, \delta_{j}<0$ (see (5.2)). We fix $\tau, \delta<0$ such that $|\tau|<\min \left\{\left|\tau_{j}\right|\right\}$ and $|\delta|<\min \left\{\left|\delta_{j}\right|\right\}$. By definition, $\phi \in \tilde{L}_{\tau, \delta}^{2}\left(\mathbf{R}^{2}\right)$ if each $H_{j}^{*}\left(\chi_{j} \phi\right) \in$ 
$L_{v_{j}, \tau, \delta}^{2}\left(\mathbf{R}^{2}\right)$ for $j=1, \ldots, V$ and with this definition we set

$$
\|\phi\|_{\tilde{L}_{\tau, \delta}^{2}\left(\mathbf{R}^{2}\right)}:=\sum_{j=1}^{V}\left\|H_{j}^{*}\left(\chi_{j} \phi\right)\right\|_{L_{v_{j}, \tau, \delta}^{2}\left(\mathbf{R}^{2}\right)} .
$$

Similarly, we will say that $\phi \in \tilde{W}_{\tau, \delta}^{2,2}\left(\mathbf{R}^{2}\right)$ if each $H_{j}^{*}\left(\chi_{j} \phi\right) \in W_{v_{j}, \tau, \delta}^{2,2}\left(\mathbf{R}^{2}\right)$ for $j=$ $1, \ldots, V$ and we set

$$
\|\phi\|_{\tilde{W}_{\tau, \delta}^{2,2}\left(\mathbf{R}^{2}\right)}:=\sum_{j=1}^{V}\left\|H_{j}^{*}\left(\chi_{j} \phi\right)\right\|_{W_{v_{j}, \tau, \delta}^{2,2}\left(\mathbf{R}^{2}\right)} .
$$

We also need cutoff functions which are subordinate to the rays $R_{1}, \ldots, R_{2 L}$ associated to the network defined by $\Lambda_{1}, \ldots, \Lambda_{L}$. To do so, we choose $\rho>0$ large enough such that the open disc $D_{\rho}$ of radius $\rho$ contains all the vertices $\mathrm{x}_{1}, \ldots, \mathrm{x}_{V}$ and we choose $\tilde{\eta}>0$ small enough. For each ray $R_{j}$, we define a cutoff function $\tilde{\chi}_{j}$ which is identically equal to 1 in

$$
\begin{aligned}
\tilde{O}_{j}:=\left\{\mathrm{x} \in \mathbf{R}^{2}:|\mathrm{x}| \geq \rho\right. \\
\left.\quad \text { and } \operatorname{dist}\left(\mathrm{x}, R_{j}\right) \leq \operatorname{dist}\left(\mathrm{x}, R_{k}\right), \quad \text { for } \quad k=1, \ldots, 2 L, \quad k \neq j\right\},
\end{aligned}
$$

and identically equal to 0 in

$$
\left\{\mathrm{x} \in \mathbf{R}^{2}: \operatorname{dist}\left(\mathrm{x}, \tilde{O}_{j}\right) \geq \tilde{\eta}\right\} .
$$

We also assume that

$$
\left\|\tilde{\chi}_{j}\right\|_{\mathcal{C}^{\infty}\left(\mathbf{R}^{2}\right)} \leq C
$$

for all $j=1, \ldots, 2 L$.

Without loss of generality, we can assume that none of the $\Lambda_{j}$ is parallel to the $x$-axis. We define the $4 L$-dimensional deficiency space

$$
\tilde{\mathfrak{D}}_{\epsilon}:=\bigoplus_{j=1, \ldots, 2 L} \operatorname{Span}\left\{\tilde{\chi}_{j} \partial_{x} \tilde{u}_{\epsilon}, \tilde{\chi}_{j}\left(x \partial_{y}-y \partial_{x}\right) \tilde{u}_{\epsilon}\right\} .
$$

The fact that none of the lines $\Lambda_{j}$ is parallel to the $x$-axis guaranties that $\partial_{x} \tilde{u}_{\epsilon}$ does not decay exponentially fast to 0 at infinity and

$$
\tilde{L}_{\tau, \delta}^{2}\left(\mathbf{R}^{2}\right) \cap \tilde{\mathfrak{D}}_{\epsilon}=\emptyset .
$$

We define

$$
\begin{array}{ccc}
\tilde{\mathcal{L}}_{\epsilon}: \quad \tilde{W}_{\tau, \delta}^{2,2}\left(\mathbf{R}^{2}\right) \oplus \tilde{\mathfrak{D}}_{\epsilon} & \rightarrow \tilde{L}_{\tau, \delta}^{2}\left(\mathbf{R}^{2}\right) \\
\phi & \mapsto \quad \tilde{L}_{\epsilon} \phi
\end{array}
$$

where

$$
\tilde{L}_{\epsilon}:=\epsilon^{2} \Delta+1-3 \tilde{u}_{\epsilon}^{2}
$$

In the rest of the paper, we will use $c$ and $C$ to denote general constants which do not depend on $\epsilon$ and will change from step to step.

Taking advantage of the fact that, near each vertex $\mathbf{x}_{j}$, the function $\tilde{u}_{\epsilon}$ is equal to $u_{j}$, we will use the result of Proposition 5.2 to prove the :

Proposition 6.1. Assume that the solutions $v_{j}$ used to construct $\tilde{u}_{\epsilon}$ are all regular. For all $\tau, \delta<0$ close enough to 0 , there exists $\epsilon_{0}>0$ such that, for all $\epsilon \in\left(0, \epsilon_{0}\right)$, the linear operator $\tilde{\mathcal{L}}_{\epsilon}$ has a right inverse

$$
\tilde{\mathcal{G}}_{\epsilon}: \tilde{L}_{\tau, \delta}^{2}\left(\mathbf{R}^{2}\right) \rightarrow \tilde{W}_{\tau, \delta}^{2,2}\left(\mathbf{R}^{2}\right) \oplus \tilde{\mathfrak{D}}_{\epsilon},
$$


whose norm is bounded by a constant (independent of $\epsilon$ ) times $\epsilon^{1-V} e^{-\frac{\tau \ell}{\epsilon}}$, where $\ell$ is the maximum of the lengths of the edges of the network defined by $\Lambda_{1}, \ldots, \Lambda_{L}$.

Proof. We would like to solve

$$
\tilde{L}_{\epsilon} w=f
$$

where $f \in \tilde{L}_{\tau, \delta}^{2}\left(\mathbf{R}^{2}\right)$. Let us denote

$$
F_{j}:=H_{j}^{*}\left(\chi_{j} f\right) .
$$

Thanks to the result of Proposition 5.1, we can solve

$$
L_{v_{j}} \stackrel{\circ}{W}_{j}=F_{j},
$$

where $\stackrel{\circ}{W}_{j} \in W_{v_{j}, \tau, \delta}^{2,2}\left(\mathbf{R}^{2}\right) \oplus \mathfrak{D}_{v_{j}}$. Observe that there is no uniqueness in the definition of $\stackrel{\circ}{W}_{j}$ since, as explained in the previous section, the operator $\mathcal{L}_{v_{j}}$ has a 4-dimensional kernel and we can freely add a linear combination of the elements of the kernel to $\stackrel{\circ}{W}_{j}$. Decomposing

$$
\mathfrak{D}_{v_{j}}={\stackrel{\mathfrak{D}}{v_{j}}} \oplus \operatorname{Ker} \mathcal{L}_{v_{j}}
$$

we can write in general:

$$
\stackrel{\circ}{W}_{j}=\stackrel{\circ}{V}_{j}+\stackrel{\circ}{D}_{j}+\check{\check{D}}_{j},
$$

where ${\stackrel{\circ}{V_{j}}}_{j} \in W_{v_{j}, \tau, \delta}^{2,2}\left(\mathbf{R}^{2}\right)$ and $\stackrel{\circ}{D}_{j} \in \stackrel{\circ}{\mathfrak{D}}_{v_{j}}, \check{D}_{j} \in \operatorname{Ker} \mathcal{L}_{v_{j}}$. Moreover, we have, again by Proposition 5.1:

$$
\left\|\stackrel{\circ}{V}_{j}+\stackrel{\circ}{D}_{j}\right\|_{W_{v_{j}, \tau, \delta}^{2,2}\left(\mathbf{R}^{2}\right) \oplus \mathcal{D}_{v_{j}}} \leq C\left\|F_{j}\right\|_{L_{v_{j}, \tau, \delta}^{2}\left(\mathbf{R}^{2}\right)}
$$

We will now explain how to choose the correct solutions of (6.2) to construct a solution of $\tilde{L}_{\epsilon} w=f$. The strategy follows the line of the construction of the approximate solution and hence we keep the notations used in the construction of $\tilde{u}_{\epsilon}$. We proceed inductively starting from the vertex whose $y$-coordinate is the largest. In fact, we choose a solution of (6.2) for $j=1$ with $\check{D}_{j} \equiv 0$ so that $\stackrel{\circ}{W}_{1}=\stackrel{\circ}{V}_{1}+\stackrel{\circ}{D}_{1}$ and we define

$$
w_{1}:=K_{1}^{*} \stackrel{\circ}{W}_{1}
$$

Note that by (6.3) we have:

$$
\left\|\stackrel{\circ}{W}_{1}\right\|_{W_{v_{1}, \tau, \delta}^{2,2}\left(\mathbf{R}^{2}\right) \oplus \mathfrak{D}_{v_{1}}} \leq C\left\|F_{1}\right\|_{L_{v_{1}, \tau, \delta}^{2}\left(\mathbf{R}^{2}\right)} .
$$

Let us assume that we have already constructed $w_{1}, \ldots, w_{k-1}$ and let us explain how to construct $w_{k}=K_{k}^{*} \stackrel{\circ}{W}_{k}$. Three cases have to be distinguished exactly as in the construction of $\tilde{u}$.

Case 1. The first case corresponds to the situation where the two affine lines meeting at $\mathrm{x}_{k}$ do not contain any of the vertices $\mathrm{x}_{1}, \ldots, \mathrm{x}_{k-1}$, in which case we choose a solution to $(6.2)$ for $j=k$ with $\check{D}_{k} \equiv 0$ so that $\stackrel{\circ}{W}_{k}=$ $\stackrel{\circ}{V}_{k}+\stackrel{\circ}{D}_{k}$ and set:

$$
w_{k}:=K_{k}^{*} \stackrel{\circ}{W}_{k} .
$$

Just like in the first step we have

$$
\left\|\stackrel{\circ}{W}_{k}\right\|_{W_{v_{k}, \tau, \delta}^{2,2}\left(\mathbf{R}^{2}\right) \oplus \mathfrak{D}_{v_{k}}} \leq C\left\|F_{k}\right\|_{L_{v_{k}, \tau, \delta}^{2}\left(\mathbf{R}^{2}\right)} .
$$


Case 2. The second case corresponds to the situation where one of the lines meeting at $\mathrm{x}_{k}$ contains some of the vertices $\mathrm{x}_{1}, \ldots, \mathrm{x}_{k-1}$ while the other line does not contain any of these vertices. Let us denote by $\mathrm{x}_{j}$ the vertex in the collection $\mathrm{x}_{1}, \ldots, \mathrm{x}_{k-1}$ which belongs to one of the affine lines meeting at $\mathrm{x}_{k}$ and which is the closest to $\mathrm{x}_{k}$. Recall that we have arranged things in such a way that one of the ends of $u_{j}$ coincides with one of the ends of $u_{k}$, and asymptotically these ends are parallel to the edge $\left[\mathrm{x}_{j}, \mathrm{x}_{k}\right]$. To fix the ideas let us assume that these ends correspond to the ends of $v_{j}$ and $v_{k}$ which are in $Q^{\llcorner}$and let us pay some attention about the behavior of both $\stackrel{\circ}{W}_{j}$ and $\stackrel{\circ}{W}_{k}$ in $Q^{\llcorner}$.

We choose an element $\check{D}_{k}$ in the kernel of $\mathcal{L}_{v_{k}}$ so that, $K_{j}^{*}\left(\stackrel{\circ}{D}_{j}+\check{D}_{j}\right)$ and $K_{k}^{*}\left(\check{D}_{k}+\check{D}_{k}\right)$ have the same behavior along the end parallel to the edge $\left[\mathrm{x}_{j}, \mathrm{x}_{k}\right]$. We will see that this is possible thanks to the result of Proposition 5.2. To make things more precise, let us assume that the ends parallel to the edge $\left[\mathrm{x}_{j}, \mathrm{x}_{k}\right]$ along which we try to connect the different solutions are included in the $x$-axis and respectively coincide with the half line $(-b,+\infty)$ and $(-\infty, b)$. Then, in a neighborhood of the origin, the function $K_{j}^{*}\left(\stackrel{\infty}{D}_{j}\right)$ can be expanded as

$$
\operatorname{sech}^{2}\left(\frac{y}{\sqrt{2} \epsilon}\right)\left(\stackrel{\circ}{\kappa}, j_{\mathbf{t}}+\stackrel{\circ}{\kappa} \mathbf{r}, j \frac{x+b}{\epsilon}\right)+\mathcal{O}\left(e^{-\frac{c}{\epsilon}}\right)
$$

for some $\stackrel{\circ}{\kappa} \mathbf{t}, j, \stackrel{\kappa}{r}, j_{\mathbf{r}} \in \mathbf{R}$, while the function $K_{k}^{*}\left(\stackrel{\circ}{D}_{k}\right)$ can be expanded as

$$
\operatorname{sech}^{2}\left(\frac{y}{\sqrt{2} \epsilon}\right)\left(\check{\kappa}_{\mathbf{t}, k}+\stackrel{\circ}{\kappa} \mathbf{r}, k_{\frac{x-b}{\epsilon}}\right)+\mathcal{O}\left(e^{-\frac{c}{\epsilon}}\right),
$$

for some $\stackrel{\circ}{\kappa} \mathbf{t}, k, \stackrel{\kappa}{\mathbf{r}, k}_{\mathbf{r}} \in \mathbf{R}$ and for some constant $c>0$. Thanks to the result of Proposition 5.2, it is possible to find an a function $\check{D}_{k} \in \operatorname{Ker} \mathcal{L}_{v_{k}}$ whose coefficients are given by

$$
\kappa_{\mathbf{t}, k}^{\llcorner}=\stackrel{\circ}{\kappa}_{\mathbf{t}, j}-\stackrel{\circ}{\kappa}_{\mathbf{t}, k}+\frac{2 b \stackrel{\circ}{\kappa}_{\mathbf{r}, j}}{\epsilon},
$$

and

$$
\kappa_{\mathbf{r}, k}^{\llcorner}=\stackrel{\circ}{\kappa}_{\mathbf{r}, j}-\stackrel{\circ}{\kappa}_{\mathbf{r}, k},
$$

so that $K_{k}^{*}\left(\check{D}_{k}\right)$ has the following expansion

$$
\operatorname{sech}^{2}\left(\frac{y}{\sqrt{2} \epsilon}\right)\left(\stackrel{\circ}{\kappa}_{\mathbf{t}, j}-\stackrel{\circ}{\kappa}_{\mathbf{t}, k}+\frac{2 b \stackrel{\circ}{\kappa}_{\mathbf{r}, j}}{\epsilon}+\left(\stackrel{\circ}{\kappa}_{\mathbf{r}, j}-\stackrel{\circ}{\kappa}_{\mathbf{r}, k}\right) \frac{x-b}{\epsilon}\right)+\mathcal{O}\left(e^{-\frac{c}{\epsilon}}\right),
$$

close to the origin. We then define

$$
w_{k}:=K_{k}^{*}\left(\stackrel{\circ}{W}_{k}+\check{D}_{k}\right)
$$

For future use, observe that

$$
\left\|\stackrel{\circ}{W}_{k}\right\|_{W_{v_{k}, \tau, \delta}^{2,2}\left(\mathbf{R}^{2}\right) \oplus \mathfrak{D}_{v_{k}}} \leq C\left\|F_{k}\right\|_{L_{v_{k}, \tau, \delta}^{2}\left(\mathbf{R}^{2}\right)},
$$

and we also have

$$
\left\|\check{D}_{k}\right\|_{W_{v_{k}, \tau, \delta}^{2,2}\left(\mathbf{R}^{2}\right) \oplus \mathfrak{D}_{v_{k}}} \leq \frac{C}{\epsilon}\left(\left|\stackrel{\circ}{\mathbf{r}, j}_{\mathbf{r}}\right|+|\stackrel{\kappa}{\mathbf{t}, j}|+\left\|F_{k}\right\|_{L_{v_{k}, \tau, \delta}^{2}\left(\mathbf{R}^{2}\right)}\right) .
$$


Case 3. The third case we need to consider corresponds to the situation where both affine lines meeting at $\mathrm{x}_{k}$ contain some of the vertices $\mathrm{x}_{1}, \ldots, \mathrm{x}_{k-1}$. Let us denote by $\mathrm{x}_{j}$ and $\mathrm{x}_{j^{\prime}}$ the vertices in the collection $\mathrm{x}_{1}, \ldots, \mathrm{x}_{k-1}$ which belong to the two affine lines meeting at $\mathrm{x}_{k}$ and which are the closest to $x_{k}$. Recall that we have arranged things in such a way that one of the ends of $u_{j}$ coincides with one of the ends of $u_{k}$ and one of the ends of $u_{j^{\prime}}$ coincides with another end of $u_{k}$. Observe, and this is a key point, that the prescribed ends of $u_{k}$ are always contained in a half plane (this follows from the fact that the $y$-coordinate of $\mathrm{x}_{j}$ and $\mathrm{x}_{j^{\prime}}$ are larger than the $y$-coordinate of $\mathrm{x}_{k}$ ). This allows us to use the results of Proposition 5.2 in what follows.

To fix the ideas let us assume that the ends of $u_{k}$ correspond to the ends of $v_{k}$ which are in upper half space while the ends of $u_{j}$ and $u_{j^{\prime}}$ correspond to the ends of $v_{j}$ and $v_{j^{\prime}}$ which are in $Q^{\llcorner}$. Repeating the argument presented above in Case 2 simultaneously for the two ends which are parallel respectively to the segments $\left[\mathrm{x}_{k}, \mathrm{x}_{j}\right]$ and $\left[\mathrm{x}_{k}, \mathrm{x}_{j^{\prime}}\right]$, we can determine the coefficients $\kappa_{\mathbf{t}, k}^{\llcorner}, \kappa_{\mathbf{r}, k}^{\llcorner}$and $\kappa_{\mathbf{t}, k}^{\lrcorner}, \kappa_{\mathbf{r}, k}^{\lrcorner}$by formula which are similar to the ones used in Case 2 and then use the result of Proposition 5.2 to find $\check{D}_{k} \in \operatorname{Ker} \mathcal{L}_{v_{k}}$ whose asymptotic behavior in the upper half plane is prescribed by these coefficients. It is at this point in the proof that we use the fact that the model 4-ended solutions used to construct the approximate solution $\tilde{u}_{\epsilon}$ are regular.

As in Case 2, we then define

$$
w_{k}:=K_{k}^{*}\left(\stackrel{\circ}{W}_{k}+\check{D}_{k}\right)
$$

We note that the estimate analogous to (6.6) holds and, this time, we find

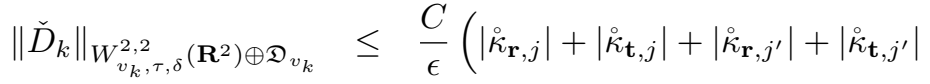

$$
\begin{aligned}
& \left.+\left\|F_{k}\right\|_{L_{v_{k}, \tau, \delta}^{2}\left(\mathbf{R}^{2}\right)}\right) \text {. }
\end{aligned}
$$

Step 3. To complete the construction of the approximate solution, we use appropriate cutoff functions to glue together $w_{1}, \ldots, w_{V}$ at the different vertices. We have already introduced the partition of unity $\chi_{1}, \ldots, \chi_{V}$. Let us now define the cutoff functions $\dot{\circ}_{1}, \ldots, \stackrel{\circ}{\chi}_{V}$ by the fact that $\dot{\circ}_{j}$ is identically equal to 1 in the union of those domains among $\Omega_{1}, \ldots, \Omega_{D}$ which have the property that $\mathrm{x}_{j}$ belongs to the closure of at least one of them. We also ask that $\dot{\circ}_{j}$ is identically equal to 0 in the complement of the set of points at distance less than $\eta$ from the union of those domains among $\Omega_{1}, \ldots, \Omega_{D}$ which have the property that $\mathrm{x}_{j}$ belongs to the closure of at least one of them. We finally ask that

$$
\left\|\stackrel{\circ}{\chi}_{j}\right\|_{\mathcal{C}^{\infty}\left(\mathbf{R}^{2}\right)} \leq C
$$

With these definitions at hand, the approximate solution $w$ is defined by

$$
w:=\sum_{j=1}^{V} \chi_{j} K_{j}^{*}\left(\stackrel{\circ}{D}_{j}+\check{D}_{j}\right)+\sum_{j=1}^{V} \stackrel{\circ}{\chi}_{j} K_{j}^{*} \stackrel{\circ}{V}_{j}
$$


Following the construction step by step and using the definition of the weighted spaces, it is easy to check that

$$
\|w\|_{\tilde{L}_{\tau, \delta}^{2}\left(\mathbf{R}^{2}\right) \oplus \tilde{\mathfrak{D}}_{\epsilon}} \leq C \epsilon^{1-V} e^{-\frac{\tau \ell}{\epsilon}}\|f\|_{\tilde{L}_{\tau, \delta}^{2}\left(\mathbf{R}^{2}\right)},
$$

where $\ell$ is the maximum of the lengths of the edges of the network defined by $\Lambda_{1}, \ldots, \Lambda_{L}$. Indeed, this follows by induction from the results of Proposition 5.1 and from estimates (6.4), (6.5) and (6.6) and their analogs in Case 3. Note that since $\tau$ and $\delta$ are negative, the coefficient in (6.7) before the norm of $f$ is actually very large. We now claim that there holds

$$
\left\|L_{\tilde{u}_{\epsilon}} w-f\right\|_{\tilde{L}_{\tau, \delta}^{2}\left(\mathbf{R}^{2}\right)} \leq C e^{-\frac{c}{\epsilon}}\|f\|_{\tilde{L}_{\tau, \delta}^{2}\left(\mathbf{R}^{2}\right)} .
$$

Accepting this last claim, since $c>0$, the result follows from a standard perturbation argument, provided $\epsilon>0$ is taken close enough to 0 .

It remains to prove estimate (6.8). Keep in mind that $\tau, \delta$ are close enough to 0 . First of all, given $k=1, \ldots, V$, since $\tilde{u}_{\epsilon}$ is equal to one of the model 4-ended solutions around each vertex, it is not hard to see that

$$
H_{k}^{*}\left(\chi_{k} \tilde{L}_{\varepsilon} w\right)=H_{k}^{*} \chi_{k} L_{v_{k}}\left(H_{k}^{*} w\right)+\mathcal{O}\left(e^{-\frac{c}{\varepsilon}}\right) w .
$$

Let us define $D_{j}:=\stackrel{\circ}{D}_{j}+\check{D}_{j}$, for $j=1, \ldots, V$. By definition of $w$ we have

$$
L_{v_{k}}\left(H_{k}^{*} w\right)=L_{v_{k}}\left(\stackrel{\circ}{V}_{k} H_{k}^{*} \stackrel{\circ}{\chi}_{k}\right)+L_{v_{k}}\left(D_{k} H_{k}^{*} \chi_{k}\right)+\sum_{j \neq k}\left(L_{v_{k}}\left(\stackrel{\circ}{V}_{j} H_{k}^{*} \stackrel{\circ}{\chi}_{j}\right)+L_{v_{k}}\left(D_{j} H_{k}^{*} \chi_{j}\right)\right) .
$$

By construction $\chi_{k} \stackrel{\circ}{\chi}_{k}=\chi_{k}$, hence we get

$$
\left(H_{k}^{*} \chi_{k}\right)\left(L_{v_{k}}\left(\stackrel{\circ}{V}_{k} H_{k}^{*} \stackrel{\circ}{\chi}_{k}\right)+L_{v_{k}}\left(D_{k} H_{k}^{*} \chi_{k}\right)\right)=F_{k}+H_{k}^{*} \chi_{k} L_{v_{k}}\left(D_{k} H_{k}^{*}\left(\chi_{k}-1\right)\right) .
$$

In Supp $\chi_{k}\left(1-\chi_{k}\right) \cap\left(\mathbf{R}^{2} \backslash B_{\rho}\right)$, we can estimate

$$
\begin{aligned}
\left\|H_{k}^{*} \chi_{k} L_{v_{k}}\left(D_{k} H_{k}^{*}\left(\chi_{k}-1\right)\right)\right\|_{\tilde{L}_{\tau, \delta}^{2}\left(\mathbf{R}^{2} \backslash B_{\rho}\right)} & \leq C e^{-\frac{c}{\epsilon}}\left\|D_{k}\right\|_{\mathfrak{D}_{v_{k}}} \\
& \leq C e^{-\frac{c}{\epsilon}}\|f\|_{\tilde{L}_{\tau, \delta}^{2}\left(\mathbf{R}^{2}\right)} .
\end{aligned}
$$

Inside the ball of radius $\rho$, in the set $\operatorname{Supp} \chi_{k}\left(\chi_{k}-1\right) \cap \operatorname{Supp} \chi_{j}$, when $j \neq k$, we make use of the fact that the functions $D_{j}$ and $D_{k}$ have been designed appropriately so that their leading terms match along the edge $\left[\mathrm{x}_{k}, \mathrm{x}_{j}\right]$ as has been described above. Observe that in a neighborhood of this edge, we have $\chi_{j}=1-\chi_{k}$ in the set we consider and, using this, we conclude that

$$
\left\|\left(H_{k}^{*} \chi_{k}\left(\chi_{k}-1\right)\right) L_{v_{k}} D_{k}+\left(H_{k}^{*} \chi_{k} \chi_{j}\right) L_{v_{k}} D_{j}\right\|_{\tilde{L}_{\tau, \delta}^{2}\left(\mathbf{R}^{2}\right)} \leq C e^{-\frac{c}{\epsilon}}\|f\|_{\tilde{L}_{\tau, \delta}^{2}\left(\mathbf{R}^{2}\right)} .
$$

Furthermore, to estimate the term $H_{k}^{*} \chi_{k} L_{v_{k}}\left(\stackrel{\circ}{V_{j}} H_{k}^{*} \stackrel{\circ}{j}_{j}\right)$ with $j \neq k$, one uses the fact that the function $F_{j}$ is compactly supported and $\stackrel{\circ}{V}_{j}$ actually stays in a better space $W_{v_{j}, \tau_{0}, \delta_{0}}^{2,2}\left(\mathbf{R}^{2}\right)$, with some fixed constants $\delta_{0}<0$ and $\tau_{0}<0$. Other terms appearing in the expression for $H_{k}^{*} \chi_{k} L_{v_{k}} H_{k}^{*} w$ can be estimated in a similar way and the claim follows from summing up all these estimates over $k$. This completes the proof of the proposition. 


\section{ThE NONLINEAR ARGUMENT}

We have now all the tools needed to construct a solution of (2.1) by perturbing the approximate solution $\tilde{u}_{\epsilon}$. As usual, the idea is to look for a solution of the form $u=\tilde{u}_{\epsilon}+w$, where $w$ is small. This amounts to solving the following nonlinear problem

$$
\tilde{L}_{\epsilon} w+\tilde{E}_{\epsilon}+\tilde{Q}_{\epsilon}(w)=0
$$

where

$$
\tilde{L}_{\epsilon}:=\epsilon^{2} \Delta+1-3 \tilde{u}_{\epsilon}^{2}, \quad \tilde{E}_{\epsilon}:=\epsilon^{2} \Delta \tilde{u}_{\epsilon}+\tilde{u}_{\epsilon}-\tilde{u}_{\epsilon}^{3},
$$

and

$$
\tilde{Q}_{\epsilon}(w):=w^{3}+3 \tilde{u}_{\epsilon} w^{2} .
$$

Previously (see (3.5)) we mentioned that the error $\tilde{E}_{\epsilon}$ tends to 0 as $\epsilon$ tends to 0 and we have also analyzed the invertibility of the linear operator which appears in this nonlinear problem. As is clear from the analysis of the previous section, the right inverse of the operator $\tilde{\mathcal{L}}_{\epsilon}$ obtained in Proposition 6.1 , takes values in $\tilde{W}_{\tau, \delta}^{2,2}\left(\mathbf{R}^{2}\right) \oplus \tilde{\mathfrak{D}}_{\epsilon}$ and the functions in this space do not necessarily decay exponentially fast at infinity. Obviously, this fact causes some trouble in the solvability of a nonlinear problem and to overcome this issue we need to modify the nonlinear problem we consider.

By definition, $w \in \tilde{W}_{\tau, \delta}^{2,2}\left(\mathbf{R}^{2}\right) \oplus \tilde{\mathfrak{D}}_{\epsilon}$ is the sum of a function of $\tilde{w} \in \tilde{W}_{\tau, \delta}^{2,2}\left(\mathbf{R}^{2}\right)$ and a function

$$
\stackrel{\circ}{w}=\sum_{j=1}^{R} \stackrel{\circ}{w}_{j}
$$

where $\stackrel{\circ}{w}_{j}$ is in turn are linear combinations of $\epsilon \tilde{\chi}_{j} \partial_{x} \tilde{u}_{\epsilon}$ and $\tilde{\chi}_{j}\left(x \partial_{y}-y \partial_{x}\right) \tilde{u}_{\epsilon}$. We can then define $\kappa_{\mathbf{t}, j}$ and $\kappa_{\mathbf{r}, j}$ to be the coefficients of the decomposition of $H_{j}^{*} \stackrel{\leftrightarrow}{j}_{j}$ as in (5.5) (observe that in this decomposition, at most one of the couple $\left(\kappa_{\mathbf{t}}^{\bullet}, \kappa_{\mathbf{r}}^{\bullet}\right)$ is not identically equal to 0$)$. We define a diffeomorphism associated to $w$ by the formula

$$
\Phi_{\mathfrak{w}}(\mathrm{x})=\mathrm{x}+\sum_{j=1}^{R} \tilde{\chi}_{j}(\mathrm{x})\left(\mathrm{x}_{k_{j}}+\mathcal{R}_{-\kappa_{\mathbf{r}, j}}\left(\mathrm{x}-\mathrm{x}_{k_{j}}\right)-\epsilon \kappa_{\mathbf{t}, j} \mathrm{a}_{k_{j}}\right)
$$

where $\mathrm{x}_{k_{j}}$ is the vertex associated to the ray $R_{j}$ and

$$
\mathrm{a}_{k_{j}} \cdot \mathrm{x}+b_{k_{j}}=0
$$

the equation of the line containing the ray $R_{j}$ and, as usual, $\mathcal{R}_{\theta}$ is the rotation by angle $\theta$ in the plane. Geometrically this diffeomorphism corresponds to a rotation and a translation of each ray.

Given the above decomposition of $w \in \tilde{W}_{\tau, \delta}^{2,2}\left(\mathbf{R}^{2}\right) \oplus \tilde{\mathfrak{D}}_{\epsilon}$, we then look for a solution of (2.1) of the form

$$
u:=\left(\tilde{u}_{\epsilon}+\tilde{w}\right) \circ \Phi_{\grave{w}}^{-1},
$$

and we solve, instead of (2.1),

$$
\left(\epsilon^{2} \Delta\left(\left(\tilde{u}_{\epsilon}+\tilde{w}\right) \circ \Phi_{\dot{w}}^{-1}\right)\right) \circ \Phi_{\mathscr{w}}+\left(\tilde{u}_{\epsilon}+\tilde{w}\right)+\left(\tilde{u}_{\epsilon}+\tilde{w}\right)^{3}=0 .
$$

A comment about the form of this problem is due. Indeed, at first glance, it would look more natural to define $u=\left(\tilde{u}_{\epsilon}+\tilde{w}\right) \circ \Phi_{\tilde{w}}$ instead of $u=\left(\tilde{u}_{\epsilon}+\tilde{w}\right) \circ \Phi_{\dot{w}}^{-1}$. However, it turns out that, with the latter choice the expression of $\left(\Delta\left(\left(\tilde{u}_{\epsilon}+\tilde{w}\right) \circ \Phi_{\dot{w}}^{-1}\right)\right) \circ \Phi_{\tilde{w}}$ is simpler and this follows from the observation that, for functions of one variable, 
$\left(u \circ f^{-1}\right)^{\prime} \circ f=u^{\prime} / f^{\prime}$ does not involve any composition with $f^{-1}$ while $(u \circ f)^{\prime} \circ f^{-1}=$ $u^{\prime} f^{\prime} \circ f^{-1}$ does involve some composition of $f^{\prime}$ and $f^{-1}$.

We have the following:

Lemma 7.1. Assume that $\tau, \delta<0$ are fixed close enough to 0 . Then, there exist constants $C>0$ and $c_{*}, \tilde{\ell}>0$ such that

$$
\left\|\epsilon^{2} \Delta \tilde{u}_{\epsilon}+\tilde{u}_{\epsilon}-\tilde{u}_{\epsilon}^{3}\right\|_{\tilde{W}_{\tau, \delta}^{2,2}\left(\mathbf{R}^{2}\right)} \leq C e^{-\left(c_{*}+\tau\right) \frac{\tilde{\ell}}{\epsilon}}
$$

where $c_{*}$ does not depend on $\epsilon, \tau$ and $\delta$.

Proof. The proof of this lemma uses the simple fact that along each end, any 4ended solution converges exponentially fast to a heteroclinic solution. The estimate depends on the exponential rate at which the functions $v_{j} \in \mathcal{M}_{4}^{\text {even }}$, which are used to construct $\tilde{u}_{\epsilon}$, approach the heteroclinic solutions along their ends. This is reflected in the constant $c_{*}>0$ which only depends on this exponential rate. The estimate also depends on the cutoff functions $\eta_{j}$ and this is reflected in the fact that the constant $\tilde{\ell}>0$ only depends on the minimum of the distances between the vertex $\mathrm{x}_{j}$ and $\partial O_{j}$, for $j=1, \ldots, V$.

We now prove our main existence theorems.

Proof of Theorem 2.1 and Theorem 2.2. To begin with, let us assume that the collection of affine lines $\Lambda_{1}, \ldots, \Lambda_{L}$ is chosen in such a way that the 4-ended solutions $v_{j}$ used in the construction of the approximate solution are all regular.

We also choose $\tau, \delta<0$ close enough to 0 . In fact, we choose

$$
-c_{*} \frac{\tilde{\ell}}{\ell+\tilde{\ell}}<\tau<0
$$

where $c_{*}$ and $\tilde{\ell}$ are the constants which appear in Lemma 7.1 and $\ell$ is the one defined in Proposition 6.1.

We will prove that, for all $\epsilon$ small enough it is possible to find a (small) function $w \in \tilde{W}_{\tau, \delta}^{2,2}\left(\mathbf{R}^{2}\right) \oplus \tilde{\mathfrak{D}}_{\epsilon}$ which is a solution of (7.1).

We define

$$
N^{\epsilon}(w):=\left(\epsilon^{2} \Delta\left(\left(\tilde{u}_{\epsilon}+\tilde{w}\right) \circ \Phi_{\tilde{w}}^{-1}\right)\right) \circ \Phi_{\tilde{w}}+\left(\tilde{u}_{\epsilon}+\tilde{w}\right)+\left(\tilde{u}_{\epsilon}+\tilde{w}\right)^{3},
$$

and we rewrite the equation to solve as

$$
N^{\epsilon}(0)+D N_{\left.\right|_{0}}^{\epsilon}(w)+\int_{0}^{1}\left(D N_{\left.\right|_{s w}}^{\epsilon}-D N_{\left.\right|_{0}}^{\epsilon}\right)(w) d s=0 .
$$

The first term on the left $N^{\epsilon}(0)$ is the one which has been estimated in Lemma 7.1. The second term on the left $D N_{\left.\right|_{0}}^{\epsilon}$ is the linearized operator at $w=0$ which, thanks to the result of Proposition 6.1 can be inverted. Indeed, it is clear that, restricted to $\tilde{W}_{\tau, \delta}^{2,2}\left(\mathbf{R}^{2}\right)$, we have

$$
D N_{\left.\right|_{0}}^{\epsilon}=\tilde{L}_{\epsilon} .
$$

However, $D N_{\left.\right|_{0}}^{\epsilon}$ is not exactly equal to $\tilde{L}_{\epsilon}$ since these two operators do not coincide when restricted to $\tilde{\mathfrak{D}}_{\epsilon}$. We now make this precise. 
Elementary differential calculus shows that the partial differential of $N^{\epsilon}$ with

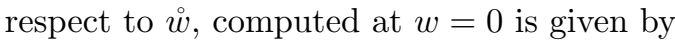

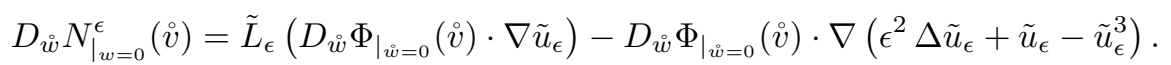

Proposition 6.1 then implies that

$$
\tilde{v}+\stackrel{\circ}{i} \rightarrow \tilde{L}_{\epsilon} \tilde{v}+\tilde{L}_{\epsilon}\left(D_{\grave{w}} \Phi_{\left.\right|_{\grave{w}=0}}(\stackrel{\circ}{)}) \cdot \nabla \tilde{u}_{\epsilon}\right)
$$

defined as an operator on $\tilde{W}_{\tau, \delta}^{2,2}\left(\mathbf{R}^{2}\right) \oplus \tilde{\mathfrak{D}}_{\epsilon}$ into $\tilde{L}_{\tau, \delta}^{2}\left(\mathbf{R}^{2}\right)$ has a right inverse whose norm is bounded by a constant times $\epsilon^{1-V} e^{-\frac{\tau \ell}{\epsilon}}$. Next, Lemma 7.1 and a perturbation argument shows that $D N_{\left.\right|_{0}}^{\epsilon}$ which defined as an operator on $\tilde{W}_{\tau, \delta}^{2,2}\left(\mathbf{R}^{2}\right) \oplus \tilde{\mathfrak{D}}_{\epsilon}$ into $\tilde{L}_{\tau, \delta}^{2}\left(\mathbf{R}^{2}\right)$ also has a right inverse whose norm is bounded by a constant times $\epsilon^{1-V} e^{-\frac{\tau \ell}{\epsilon}}$.

The existence of $w$, which is a solution of (7.2), follows at once from a standard application of a fixed point theorem for contraction mapping. We leave the details to the reader.

Let us now explain why, still in the case where the functions $v_{j} \in \mathcal{M}_{4}^{\text {even }}$ used to construct the approximate solution $\tilde{u}_{\epsilon}$ are all regular, it is possible to find solutions of (2.1) which have one end included in $\Lambda_{j}$, for each $j=1, \ldots, L$. In other words it is possible to prescribe half of the ends of the solution by requiring that each of the initial lines $\Lambda_{1}, \ldots, \Lambda_{L}$ contains an end of the solution. The idea is to modify slightly the construction of the approximate solution and the construction of the right inverse of the operator $\tilde{\mathcal{L}}_{\varepsilon}$ to ensure first that $L$ of the ends (out of the total of $2 L$ ends) of the approximate solutions $\tilde{u}_{\epsilon}$ are included in $\Lambda_{1}, \ldots, \Lambda_{L}$ and second that these ends are not modified in the perturbation argument. This last point requires that we are able to find a right inverse for $\tilde{L}_{\epsilon}$ using a $2 L$-dimensional subspace of $\tilde{\mathfrak{D}}_{\epsilon}$ which only contains the functions that are not supported in a neighborhood of the ends we try to fix.

The idea is to take advantage of the fact that, in Case 1 and Case 2 which appear in the construction of the approximate solution and in the construction of the right inverse for $\tilde{\mathcal{L}}_{\epsilon}$, we have some freedom. For instance, if we go back to the construction of the approximate solution $\tilde{u}_{\epsilon}$, we can first rotate the lines $\Lambda_{1}, \ldots, \Lambda_{L}$ such that none of them is parallel to the $x$ axis. Then, we assign 4-ended solutions to each of the vertices step by step. At each step we associate a model solution to a vertex which is chosen following some particular procedure. Then we eliminate that vertex from the list. The general principle is that for all the vertices on any fixed line, the one with larger $y$ coordinate should be eliminated earlier than the one with smaller $y$ coordinate. Note that, in this way, the approximate solution could be defined such that it has $L$ ends matching the lines $\Lambda_{1}, \ldots, \Lambda_{L}$, say matching the upper half of each line. As far as the modification of the proof of Proposition 6.1 is concerned, it can be done using a similar idea to choose the right inverse in such a way that its image maps into the direct sum of $\tilde{W}_{\tau, \delta}^{2,2}\left(\mathbf{R}^{2}\right)$ with a $2 L$-dimensional subspace of $\tilde{\mathfrak{D}}_{\epsilon}$ which only contains the functions that are not supported in a neighborhood of the ends we try to fix.

Let us observe that $\Phi_{\stackrel{\leftrightarrow}{w}}$ is defined for $\stackrel{\circ}{w} \in \tilde{\mathfrak{D}}_{\epsilon}$ while, in the fixed point argument, we only use this map restricted to a $2 L$-dimensional subspace of $\tilde{\mathfrak{D}}_{\epsilon}$ which corresponds to the image of $\tilde{\mathcal{G}}_{\epsilon}$. This is reflected in the fact that the diffeomorphism $\Phi_{\mathfrak{w}}$ does not move the $L$ ends which we have decided to fix. 
To complete the proof, let us now explain what needs to be changed if, in the construction of the approximate solution, the functions $v_{j} \in \mathcal{M}_{4}^{\text {even }}$ are not all regular. In this case, we consider a sequence of affine lines $\left(\Lambda_{1}^{(n)}\right)_{n \geq 0}, \ldots,\left(\Lambda_{L}^{(n)}\right)_{n \geq 0}$ which converge to $\Lambda_{1}, \ldots, \Lambda_{L}$ as $n$ tends to infinity. Moreover, we ask that $\Lambda_{1}^{(n)}, \ldots, \Lambda_{L}^{(n)}$ are chosen in such a way that, to construct the approximate solution $\tilde{u}_{\epsilon}^{(n)}$, one only uses 4-ended solutions which are regular. This is possible thanks to the fact that there are at most a finite number of 4 -ended solutions which are not regular. One then applies Theorem 2.1 to perturb $\tilde{u}_{\epsilon}^{(n)}$ into a solution $u_{\epsilon}^{(n)}$ of (2.1), for all $\epsilon$ small enough (say $\left.\epsilon \leq \epsilon^{(n)}\right)$. Then, $\left(u_{\epsilon^{(n)}}^{(n)}\right)_{n \geq 0}$ satisfies all the required properties.

\section{REFERENCES}

[1] F. Alessio, A. Calamai and P. Montecchiari. Saddle-type solutions for a class of semilinear elliptic equations. Adv. Differential Equations, 12 (4) : 361-380 (2007).

[2] H. Dang, P. C. Fife and L. A. Peletier. Saddle solutions of the bistable diffusion equation. Z. Angew. Math. Phys., 43 (6) : 984-998 (1992).

[3] M. del Pino, M. Kowalczyk and F. Pacard. Moduli space theory for the Allen-Cahn equation in the plane, Trans. Amer. Math. Soc., 365(2): 721-766 (2010).

[4] M. del Pino, M. Kowalczyk, F. Pacard and J. Wei.Multiple-end solutions to the Allen-Cahn equation in $\mathbf{R}^{2}$. J. Funct. Anal., 258 (2) : 458-503 (2010).

[5] N. Ghoussoub and C. Gui. On a conjecture of De Giorgi and some related problems. Math. Ann., 311(3) : 481-491, (1998).

[6] C. Gui. Symmetry of some entire solutions to the Allen-Cahn equation in two dimensions. J. Differential Equations, 252 (11) : 5853-5874 (2012).

[7] C. Gui. Symmetry of traveling wave solutions to the Allen-Cahn equaion in $\mathbb{R}^{2}$. Arch. Ration. Mech. Anal. 203(3): 1037-1065, (2012).

[8] M. Jleli and F. Pacard, An end-to-end construction for compact constant mean curvature surfaces. Pacific Journal of Maths, 221(1): 81-108 (2005).

[9] M. Kowalczyk, Y. Liu and F. Pacard. The space of four ended solutions to the Allen-Cahn equation on the plane. Ann. Inst. H. Poincaré Anal. Non Linéaire, 29 (5) : 761-781 (2012).

[10] M. Kowalczyk, Y. Liu and F. Pacard. The classification of four ended solutions to the AllenCahn equation on the plane. Preprint (2011).

[11] J. Ratzkin. An end-to-end gluing construction for surfaces of constant mean curvature, $\mathrm{PhD}$ dissertation, U. Washington (2001).

[12] M. Traizet. Construction de surfaces minimales en recollant des surfaces de Scherk. Ann. Inst. Fourier (Grenoble), 46 (5) : 1385-1442 (1996).

Michą Kowalczyk - Departamento de Ingeniería Matemática and Centro de Modelamiento Matemático (UMi 2807 CNRS), Universidad de Chile, Casilla 170 Correo 3, Santiago, Chile.

E-mail address: kowalczy@dim.uchile.cl

Yong Liu - Department of Mathematics and Physics, North China Electric Power University, Beijing, China.

E-mail address: liuyong@ncepu.edu.cn

Frank Pacard - Centre de Mathématiques Laurent Schwartz UmR-CNRS 7640, École Polytechnique, 91128 Palaiseau, France.

E-mail address: frank.pacard@math.polytechnique.fr

Juncheng Wei - Department of Mathematics, Chinese University of Hong Kong, Shatin, Hong Kong and Department of Mathematics, University of British Columbia, Vancouver, B.C., Canada V6T $1 Z 2$.

E-mail address: wei@math.cuhk.edu.hk 